\title{
Energy consumption dynamical models for smart factories based on subspace identification methods
}

\author{
Miguel Angel Bermeo-Ayerbe and Carlos Ocampo-Martinez, Senior Member, IEEE \\ Automatic Control Department, Universitat Politècnica de Catalunya, Institut de Robòtica i Informàtica Industrial (CSIC-UPC) \\ Barcelona, Spain. \{miguel.angel.bermeo, carlos.ocampo\}@upc.edu
}

\begin{abstract}
Given the need of implementing methodologies in industry for the reduction of the energy consumption costs, it is required to create modelling methodologies that, together with the use of new technologies, will allow identifying energy consumption models based on input-output data. These models will later be used to design a suitable model-based control strategy. In this paper, a subspace identification algorithm based on the RQ decomposition approach has been reported, which is both implemented and validated on a test-bench that emulates the energy consumption of an industrial machine within a manufacturing process. Subsequently, the resultant model fitting when using the proposed modelling methodology has been compared with different identification routines included into the MATLAB System Identification Toolbox ${ }^{\mathrm{TM}}$, showing, in general, better results for the proposed methodology in this paper, with up to almost $80 \%$ of fitting in some cases.

Index Terms-Industrial production systems, modeling, Energy consumption models, subspace identification, RQ decomposition
\end{abstract}

\section{INTRODUCTION}

After several reports on the depletion of the energy resources of the world in the 1970s [1], there was an increase in energy prices, new laws associated with environmental legal restrictions and incentives to create awareness in the industrial sector to implement energy efficiency strategies [2]. In 2016, the industry consumed $42 \%$ of the total world energy and $29 \%$ corresponds to the manufacturing industry [3]. Therefore, the search for strategies aimed at reducing energy costs, without sacrificing production or quality, while reducing environmental effects, has motivated the development of this paper, with the aim of proposing a strategy to obtain energy consumption models, for either industrial machines or production lines.

The traditional methodologies to determine a mathematical model involve an exhaustive procedure and are exclusively made for a given particular model of a machine or a device. However, this paradigm can be changed due to the boom of technology that allows monitoring and measuring the energy consumption in real-time. In this way, the opportunity to develop a methodology that eases the construction of the models based on the input-output data can be designed and implemented. This methodology should be oriented to control, with the aim of being used in the improvement of energy efficiency through the prediction of energy consumption [4], [5].
Several approaches have been proposed to create datadriven energy consumption models for industrial machines, which focus mainly on cutting/milling/machining methods, parameters and tool geometries [4], [6], [7]. Using strategies such as the finite element method (FEM), which manage to characterize the energy footprint with respect to the machine state and the material to be cut $/ \mathrm{milled} /$ machined [7]. Other approaches are specifically oriented to a process and/or material, through experimental/empirical models expressed as functions of independent process variables, dependent output response and coefficients [8], [9].The process variables are related to the spindle speed, the tool tip, the feed rate, the cutting/milling/machining depth, the material removal speed (MRR) and the processing time. Nowadays, models based on structures known as neural networks, machine learning and fuzzy logic functions are used to predict the future behavior of the machine energy consumption [10], [11]. However, those structures are not oriented to control, then the extensive knowledge about control will not be fully applicable.

Thus, the main contribution of this paper is the description and applicability of a subspace identification (Sub-ID) algorithm based on RQ decomposition, which will allow identifying energy consumption models using input-output data. Therefore, the obtained models follow, with a suitable accuracy, the dynamics evolution of a validated energy consumption profile, fact that allows to use such models for the design of different model-based control strategies such as optimal, predictive or robust controllers, e.g, the design and implementation of a real-time Model Predictive Control (MPC), it has been achieved using energy models computed through Sub-ID based on CVA weighing matrices [4], [5].

The remainder of this paper is organized as follows. In Section II, the problem statement and some preliminary considerations are defined. In Section III, an overview of the methodologies for system identification and model structures are presented. In Section IV, the subspace identification algorithm using RQ decomposition is deeply discussed. Section $\mathrm{V}$ defines the validation problem to support the feasibility of the proposed solution. In Section VI, the results with a comparative analysis are presented for the test-bench models. Finally, the main conclusions and lines of further research are drawn in Section VII. 


\section{PROBlEM STATEMENT}

In seeking to reduce costs in factories, energy consumption is one of the most significant expenses, in addition to the rise in energy prices and legal environmental restrictions [2]. This fact has motivated the development of an energy management platform oriented to smart factories with the main idea is to detect when and which devices deteriorate the energy efficiency of a machine or process line. In this way, implementing a control system to improve energy efficiency without sacrificing either production or quality is them quite convenient. Thus, it is needed to generate adequate energy consumption models to use in predictions and determining the optimal activation sequence in accordance with energy efficiency. However, the wide variety of devices with different natures and non-linearities make the traditional strategies for modeling a device using physical laws are non-viable methodologies to be implemented in a general and fast way in an industrial platform. Therefore, a methodology based on inputoutput data should be considered to generate energy models that respond to the phenomenological behavior related to the energy consumption of the devices.

Mainly in the manufacturing process, machines are constituted by synchronous and asynchronous devices. Since the periodic process of the machine only uses some devices, which are directly associated with the process and are known as synchronous devices. The asynchronous devices are auxiliary ones aimed to maintain, cooling, clean, and to guarantee the correct operation of the machine. Moreover, the activation sequence of synchronous devices is predefined within a machine period $\left(T_{m p}\right)$ and must be synchronized with other machines of the process line. On the other, asynchronous devices depend on sensors and/or timers to be either activated or deactivated, generally called peripheral devices. Therefore, both types of devices are controlled through discrete inputs, where their domain is defined as

$$
\mathbb{U} \triangleq\left\{u \in \mathbb{Z}_{\geq 0} \mid \underline{u} \leq u \leq \bar{u} \vee u=0\right\},
$$

being $\underline{u}, \bar{u} \in \mathbb{Z}_{\geq 1}$ the lower and upper bounds to activate the device, respectively, for a specific input.

Following [4], [5] to managing peripheral devices towards improving the energy efficiency of the industrial machines without sacrificing either production or quality, this paper has focused on showing and selecting an appropriate algorithm to identify energy models.

\section{THEORETICAL BACKGROUND}

The construction of mathematical models to mimic the dynamics of some systems according to the measurements of the input-output signals is extensively investigated in the methodologies of System Identification (SI), which allows to create an interface to apply the theory of control in applications of the real world [12], [13]. Focusing on black-box modeling, it is useful to fit the data of a particular mathematical structure with a given order. There are structures of linear and non-linear models; the procedure usually begins with a simple linear model structure and according to non-linearities, advance towards more complex structures. Nevertheless, linear models in many cases are sufficient to accurately describe the system dynamics. Moreover, strategies such as Linear Parameter Varying (LPV) [14] use a set of linear models to approximate nonlinear behaviors to avoid the use of nonlinear models, since these latter increase the complexity of the identification process and the controller.

In general, the SI and control community concentrates on three common linear time-invariant black-box model structures:

- Transfer Functions (TF), which are functions of complex variables, computed by SI algorithms from the number of poles and zeros and time- or frequency-domain data [15].

- ARX or AR models, which are input-output polynomial models specifying the number of samples of input and output data that will be considered in the regressor matrix (often calculated using least squares approaches) [16].

- State-space models, which are abstractions from input and output vectors to states variables in an Euclidean space [13].

Due to the wide applicability of space-state models in the design of modern control strategies, the state-space structure has been chosen to model the energy consumption of a set of devices in an industrial process. Many industrial processes have been described with great precision by this type of models. The state-space model in discrete time is defined as

$$
\begin{aligned}
x(k+1) & =A x(k)+B u(k)+\omega(k), \\
y(k) & =C x(k)+D u(k)+v(k),
\end{aligned}
$$

being $u \in \mathbb{R}^{m}, y \in \mathbb{R}^{l}$ and $x \in \mathbb{R}^{n}$ vectors at discrete time instant $k$ of the $m$ inputs, $l$ outputs and $n$ states of the system, respectively, $A \in \mathbb{R}^{n \times n}$ the system matrix that describes the dynamics of the system, $B \in \mathbb{R}^{n \times m}$ the input matrix that represents a linear transformation of the current input in the contribution to the next state, $C \in \mathbb{R}^{l \times n}$ the output matrix that describes the effect of current states on outputs, $D \in \mathbb{R}^{l \times m}$ the feedthrough (or feedforward) matrix allows modeling when there is a direct influence of the input on the measurements, $\omega \in \mathbb{R}^{n}$ and $v \in \mathbb{R}^{l}$ are non-measurable vector signals that affect the states and measures, respectively.

\section{Subspace IDENTIFICATION With RQ DECOMPOSITION}

Subspace-based system identification is a state-space identification approach that depends on the matrix decompositions and spatial vector projectors for which reliable numerical algorithms are available. Based on the robust deterministicstochastic subspace identification algorithm in [13], this section summarizes the implementation of this algorithm aimed at identifying the energy models. Given input-output data of length $N \in \mathbb{Z}_{\geq 0}$, let $u_{k} \in \mathbb{R}^{m}$ and $y_{k} \in \mathbb{R}^{l}$ be the input vector and the output vectors, respectively, with 
$k \in\{0,1,2, \ldots, N-1\}$. Thus, the following block Hankel matrix is constructed:

$$
H \triangleq\left(\begin{array}{c}
U_{0 \mid 2 i-1} \\
Y_{0 \mid 2 i-1}
\end{array}\right)
$$

with $U_{0 \mid 2 i-1} \in \mathbb{R}^{2 m i \times j}$ and $Y_{0 \mid 2 i-1} \in \mathbb{R}^{2 l i \times j}$ as input and output block Hankel matrices from row index 0 to $i-2$, and are defined as:

$$
\begin{aligned}
U_{0 \mid 2 i-1} \triangleq\left(\begin{array}{cccc}
u_{0} & u_{1} & \cdots & u_{j-1} \\
u_{1} & u_{2} & \cdots & u_{j} \\
\vdots & \vdots & \ddots & \vdots \\
u_{i-1} & u_{i} & \cdots & u_{i+j-2} \\
\hline u_{i} & u_{i+1} & \cdots & u_{i+j-1} \\
u_{i+1} & u_{i+2} & \cdots & u_{i+j} \\
\vdots & \vdots & \ddots & \vdots \\
u_{2 i-1} & u_{2 i} & \cdots & u_{2 i+j-2}
\end{array}\right), \\
\triangleq\left(\frac{U_{0 \mid i-1}}{U_{i \mid 2 i-1}}\right)=\left(\frac{U_{p}}{U_{f}}\right),
\end{aligned}
$$

where $i$ is the number of the block rows and is a user-defined index, which must be at least $i>n, j$ is the number of columns, typically equal to $N-2 i+2$ in order to use all given data measures, $U_{p} \in \mathbb{R}^{m i \times j}$ and $U_{f} \in \mathbb{R}^{m i \times j}$ are the past and future time of the inputs Hankel matrices. In the same way, the output Hankel matrix $Y_{0 \mid 2 i-1}, Y_{p} \in \mathbb{R}^{l i \times j}$ and $Y_{f} \in \mathbb{R}^{l i \times j}$ are computed. The optimal prediction of $Y_{f}$ can be expressed as a linear combination of subspace matrices

$$
\hat{Y}_{f}=L_{U_{p}} U_{p}+L_{Y_{p}} Y_{p}+L_{U_{f}} U_{f}
$$

being $L_{U_{p}} \in \mathbb{R}^{l i \times m i}, L_{Y_{p}} \in \mathbb{R}^{l i \times l i}$ and $L_{U_{f}} \in \mathbb{R}^{l i \times m i}$ the subspace matrices of the past input-output data and the future input data respectively, which solving the following least-squares problem can be computed:

$$
\min _{L_{U_{p}}, L_{U_{f}}, L_{Y_{f}}}\left\|Y_{f}-\left(\begin{array}{lll}
L_{U_{p}} & L_{U_{f}} & L_{Y_{f}}
\end{array}\right)\left(\begin{array}{c}
U_{p} \\
U_{f} \\
Y_{p}
\end{array}\right)\right\|_{F}^{2}
$$

where $\|\bullet\|$ is the Frobenius norm. Through the orthogonal projection of the row space of $Y_{f}$ onto the row space of $\left(U_{p} U_{f} Y_{p}\right)^{T}$ can be determined $\hat{Y}_{f}$, i.e.,

$$
\hat{Y}_{f}=Y_{f} /\left(\begin{array}{l}
U_{p} \\
U_{f} \\
Y_{p}
\end{array}\right)
$$

with / denoting the orthogonal projection ${ }^{1}$, which is expressed in function of the RQ decomposition as

$$
\begin{aligned}
& H=\left(\begin{array}{c}
U_{0 \mid 2 i-1} \\
Y_{0 \mid 2 i-1}
\end{array}\right)=R Q^{T}, \\
& ={ }_{m i} m i\left(\begin{array}{ccccc}
m i & m i & l i & l i & j \\
R_{11} & 0 & 0 & 0 \\
R_{21} & R_{22} & 0 & 0 \\
R_{31} & R_{32} & R_{33} & 0 \\
R_{41} & R_{42} & R_{43} & R_{44}
\end{array}\right)\left(\begin{array}{c}
Q_{1}^{T} \\
Q_{2}^{T} \\
Q_{3}^{T} \\
Q_{4}^{T}
\end{array}\right),
\end{aligned}
$$

where $H \in \mathbb{R}^{2(m+l) i \times j}, Q^{T} \in \mathbb{R}^{2(m+l) i \times j}$ is a orthogonal matrix, i.e., $Q^{T} Q=I$ and $R \in \mathbb{R}^{2(m+l) i \times 2(m+l) i}$ is a lower triangular matrix. Thus, allowing to calculate the subspace matrices

$$
\left(\begin{array}{lll}
L_{U_{p}} & L_{U_{f}} & L_{Y_{f}}
\end{array}\right) \triangleq\left(\begin{array}{lll}
R_{41} & R_{42} & R_{43}
\end{array}\right)\left(\begin{array}{ccc}
R_{11} & 0 & 0 \\
R_{21} & R_{22} & 0 \\
R_{31} & R_{32} & R_{33}
\end{array}\right)^{\dagger},
$$

being $\bullet \dagger$ the Moore-Penrose pseudo-inverse. Note that the computational process of the subpsace matrices is faster, since the matrix multiplication depends on a lower triangular matrix, whereby the subpsace matrices can be calculated using backsubstitution. Thus, with

$$
\left.\Pi=I-\left(\begin{array}{ll}
R_{21} & R_{22}
\end{array}\right)^{T}\left(\begin{array}{ll}
R_{21} & R_{22}
\end{array}\right)^{T}\right)^{\dagger},
$$

and computing the singular value decomposition (SVD) of

$$
U \Sigma V^{*}=\left(\left(\begin{array}{ll}
\left.L_{U_{p}}\left(\begin{array}{ll}
R_{11} & 0
\end{array}\right)+L_{Y_{p}}\left(\begin{array}{ll}
R_{31} & R_{32}
\end{array}\right)\right) \Pi \mid L_{Y_{p}} R_{33}
\end{array}\right),\right.
$$

being •* the conjugate transpose, $U \in \mathbb{R}^{l i \times l i}$ an real or complex unitary matrix, $\Sigma \in \mathbb{R}^{l i \times 2(m+l) i}$ a rectangular diagonal matrix with non-negative real numbers and $V \in$ $\mathbb{R}^{2(m+l) i \times 2(m+l) i}$ an real or complex unitary matrix.

By inspecting of the singular values extracted from the diagonal of $\Sigma$,

$$
\operatorname{diag}(\Sigma)=\left(\begin{array}{llll}
\sigma_{0} & \sigma_{1} & \cdots & \sigma_{l i}
\end{array}\right)^{T},
$$

the model order $n$ can be determined, i.e., due to the fact that the singular values represent the degree of information or energy that belong to each one with respect to the given data, the order can be understood as the accumulation of the highest singualar values. Furthermore, this order must capture the greatest amount of information and, at the same time, be as small as possible. A methodology to select $n$ properly depends on the following equation:

$$
\alpha=\frac{1}{\sum_{i=0}^{l i} \sigma_{i}} \sum_{i=0}^{n} \sigma_{i} \%
$$

with an order $n$ such that results in an $\alpha$ greater than $80 \%$, the resulting model will represent the data appropriately. However,

${ }^{1}$ The orthogonal projection of $A / B$ is defined as follows:

$$
A / B \triangleq A B^{T}\left(B B^{T}\right)^{\dagger} B .
$$


the results will depend on the nonlinearities between the input and output data.

Once the model order $n$ is defined, the matrix $U_{1}$ is calculated from $n$ columns of $U$, and $\Sigma_{1}$ forms $n$ rows and columns of $\Sigma$. Therefore, matrices $A$ and $C$ can be extracted from $\Theta$, being located in the first $n$ columns and $n+l$ rows, and ignoring the rest of the values. Considering the following definitions:

$$
\begin{aligned}
& \Gamma_{i}=U_{1} \Sigma_{1}^{1 / 2}, \quad \Gamma_{i-1}=\grave{\Gamma}_{i}, \\
& \Upsilon_{l} \triangleq\left(\begin{array}{ccc}
\Gamma_{i-1}^{\dagger} & \left(\begin{array}{lll}
R_{41}^{\prime} & R_{42}^{\prime} & R_{43}^{\prime}
\end{array}\right) \\
\left(R_{41[l]}\right. & R_{42[l]} & R_{43[l]}
\end{array}\right) \text {, }
\end{aligned}
$$

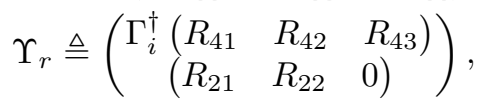

matrix $\Theta$ is then defined as

$$
\Theta=\Upsilon_{l} / \Upsilon_{r}=\left(\begin{array}{lll}
A & \phi & \cdots \\
C & \phi & \cdots
\end{array}\right)
$$

where the $\phi$ matrices are values that will be ignored, $\Gamma_{i}$ and $\Gamma_{i-1}$ are the observability matrices and the oblique projection and the Kalman filter states lead to (15) and (16), which are reported in [13]. The operators represent, $\bullet$ the matrix minus the last row, ' the matrix minus the first row and $\bullet_{[r]}$ the submatrix of row $r$ of the matrix. Finally, matrices $B$ and $D$ are computed by solving the following least-square problem:

$$
\min _{B, D}\left\|\operatorname{vec} \Psi-\left[\sum_{k=1}^{i} \Phi_{k}^{T} \otimes \Omega_{k}\right] \operatorname{vec}\left(\begin{array}{l}
D \\
B
\end{array}\right)\right\|_{F}^{2}
$$

with

$$
\begin{aligned}
\mathfrak{L} & \triangleq\left(\begin{array}{c}
A \\
\hline C
\end{array}\right) \Gamma_{i}^{\dagger}=\left(\begin{array}{lll}
\mathfrak{L}_{1 \mid 1} & \cdots & \mathfrak{L}_{1 \mid i} \\
\mathfrak{L}_{2 \mid 1} & \cdots & \mathfrak{L}_{2 \mid i}
\end{array}\right), \\
\mathfrak{M} & \triangleq \Gamma_{i-1}^{\dagger}=\left(\begin{array}{lll}
\mathfrak{M}_{1} & \ldots & \mathfrak{M}_{i-1}
\end{array}\right) \\
\Psi & \triangleq \Upsilon_{l}-\mathfrak{L}\left(\begin{array}{lll}
R_{41} & R_{42} & R_{43}
\end{array}\right) \\
\Phi & \triangleq\left(\begin{array}{llll}
R_{21} & R_{22}
\end{array}\right)=\left(\begin{array}{llll}
\Phi_{1} & \cdots & \Phi_{i}
\end{array}\right)^{T}, \\
\Omega_{1} & \triangleq\left(\begin{array}{cccc}
-\mathfrak{L}_{1 \mid 1} & \cdots & \mathfrak{M}_{i-1}-\mathfrak{L}_{1 \mid i} \\
I-\mathfrak{L}_{2 \mid 1} & \cdots & -\mathfrak{L}_{2 \mid i}
\end{array}\right)\left(\begin{array}{cc}
I & 0 \\
0 & \Gamma_{i-1}
\end{array}\right), \\
\Omega_{i} & \triangleq\left(\begin{array}{cccc}
\mathfrak{M}_{i-1}-\mathfrak{L}_{1 \mid i} & \cdots & 0 & 0 \\
-\mathfrak{L}_{2 \mid i} & \cdots & 0 & 0
\end{array}\right)\left(\begin{array}{cc}
I & 0 \\
0 & \Gamma_{i-1}
\end{array}\right),
\end{aligned}
$$

being $\otimes$ the Kronecker product, vec $\cdot$ denotes the vector operation, i.e., stacking the matrix columns on top of each other in a vector, $\mathfrak{L}_{1 \mid k}, \mathfrak{M}_{k} \in \mathbb{R}^{n \times l}, \mathfrak{L}_{2 \mid k} \in \mathbb{R}^{l \times l}, \Psi \in \mathbb{R}^{l+n \times j}$, $\Phi_{k} \in \mathbb{R}^{m \times 2 m i}$ and $\Omega_{k} \in \mathbb{R}^{m+l \times n+l}$. Note that the model computed through this algorithm will have states that satisfy the dynamics of the given input-output data, however, they do not have a direct physical interpretation.

Algorithm 1 illustrates the overall procedure of the SubID implementation explained in this section. Note that only the $R$ matrix is required and the implementation of the RQ and SVD decompositions are well-known numerical linear algebra algorithms. Therefore, this algorithm reduces the computational complexity and the memory requirements, allowing to obtain numerically robust solutions. A detailed review of this algorithm or other of Sub-ID algorithms and their implementation can be found in [13].

Algorithm 1 Subspace identification scheme

Precondition: $U$ and $Y$, the identification input-output data

1: Compute the Hankel matrix and RQ descomposition

$$
H=\left(\begin{array}{c}
U_{0 \mid 2 i-1} \\
Y_{0 \mid 2 i-1}
\end{array}\right)=R Q^{T}
$$

2: Compute the subspace matrices

$$
\left(\begin{array}{lll}
L_{U_{p}} & L_{U_{f}} & L_{Y_{f}}
\end{array}\right)=\left(\begin{array}{lll}
R_{41} & R_{42} & R_{43}
\end{array}\right)\left(\begin{array}{ccc}
R_{11} & 0 & 0 \\
R_{21} & R_{22} & 0 \\
R_{31} & R_{32} & R_{33}
\end{array}\right)^{\dagger}
$$

3: Compute the singular value descomposition

$$
\left.U \Sigma V^{*}=\left(\left(\begin{array}{ll}
L_{U_{p}}\left(R_{11}\right. & 0
\end{array}\right)+L_{Y_{p}}\left(\begin{array}{ll}
R_{31} & R_{32}
\end{array}\right)\right) \Pi \mid L_{Y_{p}} R_{33}\right)
$$

with

$$
\left.\Pi=I-\left(\begin{array}{ll}
R_{21} & R_{22}
\end{array}\right)^{T}\left(\begin{array}{ll}
R_{21} & R_{22}
\end{array}\right)^{T}\right)^{\dagger}
$$

4: Through the inspection of the singular values, the model order $n$ can be chosen and compute $U_{1}$ and $\Sigma_{1}$ from the SVD matrices

5: Determine $A$ and $C$ form first $n$ columns of $\Theta=\Upsilon_{l} / \Upsilon_{r}$, where $\Upsilon_{l}$ and $\Upsilon_{r}$ are defined as

$$
\begin{aligned}
& \Gamma_{i}=U_{1} \Sigma_{1}^{1 / 2}, \quad \Gamma_{i-1}=\grave{\Gamma}_{i} \\
& \Upsilon_{l}=\left(\begin{array}{ccc}
\Gamma_{i-1}^{\dagger} & \left(\begin{array}{lll}
R_{41}^{\prime} & R_{42}^{\prime} & R_{43}^{\prime}
\end{array}\right) \\
\left(\begin{array}{lll}
R_{41[l]} & R_{42[l]} & R_{43[l]}
\end{array}\right)
\end{array}\right) \\
& \Upsilon_{r}=\left(\begin{array}{ccc}
\Gamma_{i}^{\dagger}\left(\begin{array}{lll}
R_{41} & R_{42} & R_{43}
\end{array}\right) \\
\left(\begin{array}{lll}
R_{21} & R_{22} & 0
\end{array}\right)
\end{array}\right)
\end{aligned}
$$

6: Determine $B$ and $D$ solving the least-square problem

$$
\min _{B, D}\left\|\operatorname{vec} \Psi-\left[\sum_{k=1}^{i} \Phi_{k}^{T} \otimes \Omega_{k}\right] \operatorname{vec}\left(\begin{array}{l}
D \\
B
\end{array}\right)\right\|_{F}^{2}
$$

\section{CASE STUDY}

In order to evaluate the performance of this approach, a validation process was done on a test-bench, with devices of resistive, inductive and capacitive nature, typical in industrial machines. The test-bench has two three-phase motors, a heater and two Uninterruptible Power Supplies (UPS). The first UPS handles a fan and a lamp, and the second one two fans. Through an Alternating Current regulator (AC-R), the power consumption of each load for both UPS is controlled by a Pulse-Width Modulation (PWM) signal. That signal is generated with an Arduino Leonardo (AL) that allows to set the duty cycle between 0 and 255 levels. The other devices are activated/deactivated by a WEB LAN IP Ethernet relay controller (WLIE-RC). With a power data acquisition module, the apparent power is sensed at $4 \mathrm{kHz}$, but due to a large 
TABLE I

MODEL FITTING COMPARISON TO THE DIFFERENT STRUCTURE MODELS FOR EACH PROFILE.

\begin{tabular}{|l|c|c|ccc|ccc|}
\hline \multirow{2}{*}{ Structure $\backslash$ Profile } & $M_{p}$ & $D_{p_{3}}$ & \multicolumn{3}{c|}{$D_{p_{1}}$} & $D_{p_{2}}$ \\
& $L_{2}(\%)$ & $L_{2}(\%)$ & $L_{1}(\%)$ & $L_{2}(\%)$ & $L_{3}(\%)$ & $L_{1}(\%)$ & $L_{2}(\%)$ & $L_{3}(\%)$ \\
\hline Sub-ID (RQ) & $\mathbf{8 8 . 4 2}$ & 79.43 & $\mathbf{9 1 . 4 3}$ & $\mathbf{9 1 . 4 7}$ & $\mathbf{9 1 . 3 5}$ & $\mathbf{9 3 . 1}$ & $\mathbf{9 2 . 6 8}$ & $\mathbf{9 0 . 9 2}$ \\
MATLAB Sub-ID (CV) & 85.95 & 79.37 & 87.54 & 87.61 & 88.57 & 88.80 & 82.95 & 82.86 \\
MATLAB Sub-ID (MOESP) & 85.86 & 79.38 & 87.76 & 87.94 & 88.49 & 88.88 & 83.13 & 82.93 \\
MATLAB ARX & 87.40 & $\mathbf{8 0 . 2 0}$ & 90.32 & 89.81 & 89.35 & 89.82 & 91.48 & 90.33 \\
MATLAB ARMX & 88.32 & 80.08 & 88.85 & 88.79 & 87.37 & 88.38 & 89.39 & 87.53 \\
MATLAB SSEST & 83.04 & 73.88 & 66.09 & 66.28 & 63.51 & 60.77 & 64.26 & 54.19 \\
MATLAB TFest & 82.03 & 76.08 & 54.61 & 54.75 & 51.46 & 63.51 & 66.60 & 48.98 \\
\hline
\end{tabular}
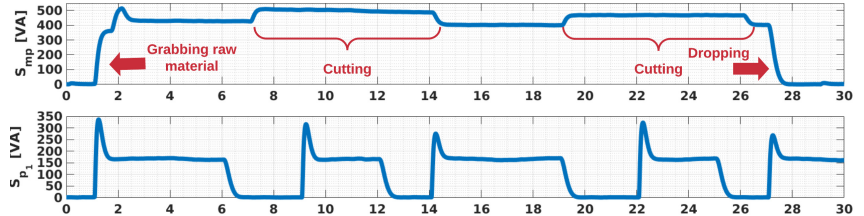

\begin{tabular}{c}
200 \\
\hline
\end{tabular}

150
$\geq 100$

2100

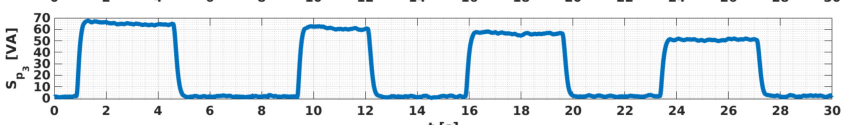

Fig. 1. Power consumption profiles for manufacturing machine and peripheral devices.

amount of data, it is re-sampled with a digital filter at $100 \mathrm{~Hz}$, since the dynamics of the devices are of $50 \mathrm{~Hz}$ nature.

The test-bench allows to emulate a manufacturing machine, where the machine receives raw material and must cut it, being assisted by three peripheral devices. The heater $\left(D_{s_{1}}\right)$ and one UPS $\left(D_{s_{2}}\right)$ are used as synchronous devices to generate the energy consumption profile of the cutting process, which consists of grabbing the raw material and making two cuts with a period of $T_{m p}=29 \mathrm{~s}$, Figure 1 shows the shape of the machine process profile. Note that most devices are singlephase, then all single-phase devices were connected to phase 2. The main process of the machine is assisted by:

(i) An air-supply pump: it provides an air stream to grip the raw material with sufficient force to ensure that the raw material does not move during cutting. The energy consumption profile is emulated by a three-phase motor $\left(D_{p_{1}}\right)$, thus the device input $u_{p_{1}}$ is defined as $u_{p_{1}} \in\{0,1\}$.

(ii) A Coolant-supply pump: by avoiding breaking the tool and deforming the raw material, the coolant system maintains the tool in the proper temperature condition. Based on a re-circulation system with a dirty and a clean tank, the dirty coolant is filtered and reused. The pump and filter systems are emulated using a three-phase motor $\left(D_{p_{2}}\right)$ and a UPS $\left(D_{p_{3}}\right)$, where the inputs are defined as $u_{p_{2}} \in\{0 \wedge 1\}$ and $u_{p_{3}} \in\{0,100,110,120,130,140\}$.

Figure 1 shows the energy consumption profile of the peripherals devices, considering different values in the inputs. For the case of $D_{p_{3}}$, the output is displayed with input step values of $110,120,130$ and 140 . In order to understand the

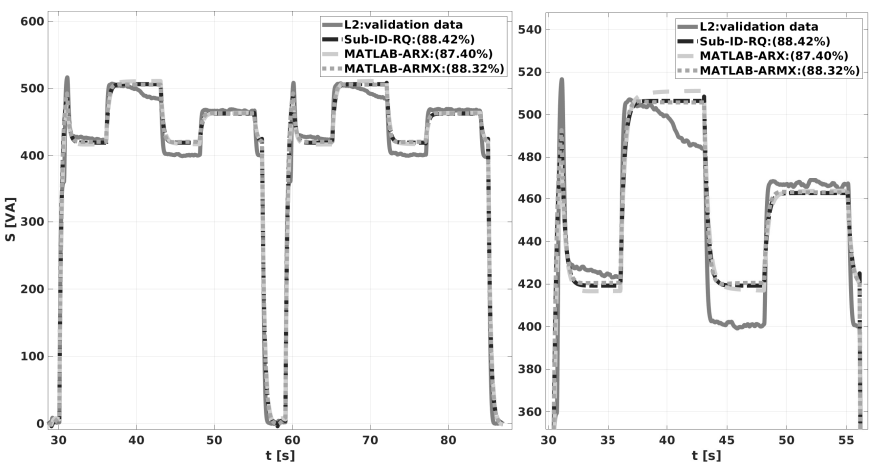

Fig. 2. Power consumption profile for manufacturing process.

Sub-ID algorithm with RQ decomposition and evaluate its performance, a first version was implemented in MATLAB and validated using this test-bench. For more details related to the experiment refer to [5].

\section{RESULTS}

Two datasets have been generated for each energy consumption profile (see Figure 1). The former is used to identify models, with $90 \mathrm{~s}$ of data, and the latter, lasting $300 \mathrm{~s}$, to validate them. Thus, the models were evaluated according to the error between both the output data from the real system and the output of the model. The following fitting function was used as a performance indicator:

$$
\gamma(Y, \hat{Y})=100\left(1-\frac{\|Y-\hat{Y}\|}{\left\|Y-\mu_{Y}\right\|}\right) \%,
$$

where $Y$ is a set of the validation output data, $\mu_{Y}$ the mean of the output data and $\hat{Y}$ the model output. Therefore, the explained Sub-ID algorithm was validated for each profile with a minimum order, and compared with other structures and implementations offered by MATLAB System Identification Toolbox ${ }^{\mathrm{TM}}$. Table I shows the results of the fitting. In general, the implemented Sub-ID offers better results with respect to others, being only slightly surpassed by the ARX and ARMX structures in $D_{p_{3}}$. Furthermore, the MATLAB Sub-ID yielded a quite large difference in fitting results with respect to the one implemented in this paper.

The performance of the best manufacturing process models are displayed in Figure 2, which demonstrates the capacity of the models to follow the profile. However, looking closer to 

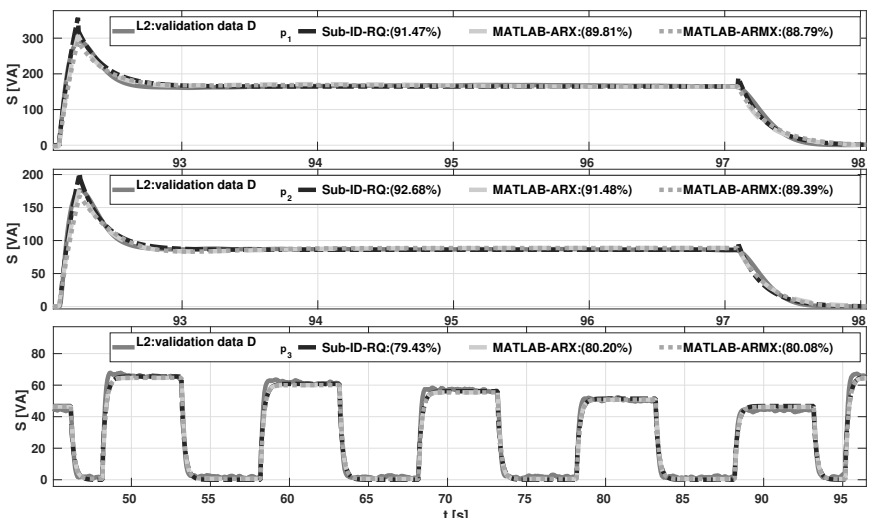

Fig. 3. Power consumption profile for peripheral devices.

the steady-state values, it can be seen how the model keeps a constant behavior while the real data show variations. Figure 3 shows the performance of the peripherals devices. The two three-phase motors have a quite small error when compared to the real data and the Sub-ID-RQ models generate highest peaks with respect to the real one. Moreover, it is worth mentioning that due to a delay between the input and output data, when the machine is turned off, the models evolution shows a slight peak. Without this delay, the model would start to drop much sooner, highly reducing the fitting. Finally, due to the non-linearities of the UPS device, the fitting of the models will vary depending on the input, being higher at lower inputs.

\section{CONCLUSIONS}

Preliminary results by using the proposed testbench demonstrate the application of identification system methods for energy consumption models, in particular using a subspace identification algorithm base on RQ decomposition. This fact, provides the ability to identify high fitting and stable models, therefore, it is useful for control paradigms. With these results, the next step is to integrate this algorithm in an adaptive strategy in order to evolve the models according to subtle changes in the system such as the wears of the tools or their replacement.

\section{ACKNOWLEDGMENT}

This work has been funded by the project IKERCON (ref. C10683).

\section{REFERENCES}

[1] G. Petrecca, Industrial Energy Management: Principles and Applications: Principles and Applications. Springer Science \& Business Media, 2012.

[2] M. Schulze, H. Nehler, M. Ottosson, and P. Thollander, "Energy management in industry-a systematic review of previous findings and an integrative conceptual framework," Journal of Cleaner Production, vol. 112, pp. 3692-3708, 2016.

[3] IEA, International energy agency: World energy statistics 2017, urlhttp://www.iea.org/statistics/, 2019.
[4] J. L. Diaz, M. Bermeo, J. Diaz-Rozo, and C. OcampoMartinez, "An optimization-based control strategy for energy efficiency of discrete manufacturing systems," ISA Transactions, 2019 (in press).

[5] M. Bermeo-Ayerbe and C. Ocampo-Martinez, "Energy efficiency improvement through mpc-based peripherals management for an industrial process test-bench," in 2019 IFAC Conference on Manufacturing Modeling, Management and Control, IFAC-PapersOnLine, 2019, pp. 1-6.

[6] L. Zhou, J. Li, F. Li, Q. Meng, J. Li, and X. Xu, "Energy consumption model and energy efficiency of machine tools: A comprehensive literature review," Journal of Cleaner Production, vol. 112, pp. 3721-3734, 2016.

[7] A. E. Santo, M. R. A. Calado, and C. M. P. Cabrita, "Dynamic simulation of electromagnetic actuators based on the co-energy map," ACES Journal, vol. 25, no. 10, pp. 1118-1128, 2010.

[8] Q. Zhong, R. Tang, J. Lv, S. Jia, and M. Jin, "Evaluation on models of calculating energy consumption in metal cutting processes: A case of external turning process," The International Journal of Advanced Manufacturing Technology, vol. 82, no. 9-12, pp. 2087-2099, 2016.

[9] W Li and S Kara, "An empirical model for predicting energy consumption of manufacturing processes: A case of turning process," Proceedings of the Institution of Mechanical Engineers, Part B: Journal of Engineering Manufacture, vol. 225, no. 9, pp. 1636-1646, 2011.

[10] HAN, Yongming, "Energy management and optimization modeling based on a novel fuzzy extreme learning machine: Case study of complex petrochemical industries," in Energy conversion and management, vol. 165, 2018, pp. 163-171.

[11] Borgia, S., Pellegrinelli, S., Bianchi, G., and Leonesio, "A reduced model for energy consumption analysis in milling," in Procedia CIRP, vol. 17, 2014, pp. 529-534.

[12] L. Ljung, "Perspectives on system identification," Annual Reviews in Control, vol. 34, no. 1, pp. 1-12, 2010.

[13] P. Overschee and B. De Moor, Subspace Identification for Linear Systems: Theory - Implementation - Applications. Boston, MA: Springer US, 1996.

[14] B. Bamieh and L. Giarre, "Identification of linear parameter varying models," International Journal of Robust and Nonlinear Control, vol. 12, no. 9, pp. 841-853, 2002.

[15] H. Garnier, M. Mensler, and A. Richard, "Continuoustime model identification from sampled data: Implementation issues and performance evaluation," International Journal of Control, vol. 76, no. 13, pp. 13371357, 2003.

[16] T. C. Hsia, System identification: least-squares methods. Lexington books Lexington, 1977, vol. 1. 